\title{
Generalization of S. Bernstein's Polynomials to the Infinite Interval
}

\section{By Otto Szasz}

$$
\text { Let, } P(u, x)=e^{-x u} \sum_{v=1}^{\infty} \frac{(u x)^{v}}{v !} f\left(\frac{v}{u}\right), u>0 \text {. }
$$

The paper studies the convergence of $P(u, x)$ to $f(x)$ as $u \rightarrow \infty$. The results obtained are generalized analogs, for the interval $0 \leq x \leq \infty$, of known properties of $\mathrm{S}$. Bernstein's approximation polynomials in a finite interval.

1. With a function $f(t)$ in the closed interval $[0,1]$, S. Bernstein in 1912 associated the polynomials

$$
B_{n}(t)=\sum_{v=0}^{n}\left(\begin{array}{l}
n \\
v
\end{array}\right) t^{v}(1-t)^{n-v} f(v / n), n=1,2,3, \cdots
$$

He proved that if $f(t)$ is continuous in the closed interval $[0,1]$, then $B_{n}(t) \rightarrow f(t)$ uniformly, as $n \rightarrow \infty$. This yields a simple constructive proof of Weierstrass's approximation theorem.

More generally the following theorems hold:

Theorem $A$. If $f(t)$ is bounded in $[0,1]$ and continuous at every point of $[a, b]$, where $0 \leqq a<$ $b \leqq 1$, then $B_{n}(t) \rightarrow f(t)$ uniformly in $[a, b]$. (See [6], p. 66) ${ }^{1}$.

Theorem $B$. If $f(t)$ is bounded in $[0,1]$ and continuous at a point $\tau$, then $B_{n}(\tau) \rightarrow F(\tau)$. (See [3], p. 112).

Theorem $C$. If $f(t)$ satisfies a Lipschitz-Hölder condition

$$
\left|f(t)-f\left(t^{\prime}\right)\right|<c\left|t-t^{\prime}\right|^{\lambda}, 0<\lambda \leqq 1,
$$

then $\left|f(t)-B_{n}(t)\right|<c_{2} n^{-\lambda / 2}, c_{1}, c_{2}$ constants (see [7], p. $53 ; 4) . \quad B_{n}(t)$ is a linear transform of the function $f(t)$; for the infinite interval $(0, \infty)$ we define an analogous transform:

$$
P(u ; f)=e^{-u x} \sum_{v=0}^{\infty} \frac{1}{v !}(u x)^{v} f(v / u), u>0 \cdot^{2} .
$$

\footnotetext{
${ }^{1}$ Figures in brackets indicate the literature references at the end of this paper.
}

We shall prove corresponding theorems of approximation for this transform; $u \rightarrow \infty$ corresponds to $n \rightarrow \infty$ in eq 1 . We also sharpen theorem $B$ to uniform convergence at the point $\tau$.

Definition: A set of continuous functions $P(u, x)$ is said to converge uniformly to the value $S$ at a point $x=\zeta$, as $u \rightarrow \infty$ if $P\left(u_{n}, x_{n}\right) \rightarrow S$, whenever $x_{n} \rightarrow \zeta$ and $u_{n} \rightarrow \infty$, as $n \rightarrow \infty$. An equivalent formulation is: to any $\epsilon>0$ there exists a $\delta(\epsilon)$ and an $\eta(\delta, \epsilon)$ so that $|P(u, x)-S|<\epsilon$ for $|x-\zeta|<\delta$ and $u>\eta$.

2. In this section we introduce some lemmas for later application.

Lemma 1. For $\lambda>0, u>0$,

$$
\sum_{|v-u| \geqq \lambda} \frac{u^{v}}{v !}<\lambda^{-2} u e^{u} .
$$

The following identity is easily verified:

$$
\sum_{v=0}^{\infty}(v-u)^{2} \frac{u^{v}}{v !}=u e^{u}
$$

it follows that

$$
\lambda^{2} \sum_{|v-u| \geqq \lambda} \frac{u^{v}}{v !}>\sum_{v=0}^{\infty}(v-u)^{2} \frac{u^{v}}{v !}=u e^{u} .
$$

This proves lemma 1.

Lemma 2. For $u \geqq 0$

$$
\sum_{0}^{\infty}|v-u| \frac{u^{v}}{v !} \leqq \sqrt{u e^{u}}
$$

${ }^{2} \mathrm{M}$. Kac also considered the transtorm (2) independently, from a similiar point of view. 
By Schwarz's inequality and by eq 4

$$
\left(\sum_{0}^{\infty}|v-u| \frac{u^{v}}{v !}\right)^{2} \leqq\left\{\sum_{0}^{\infty}(v-u)^{2} \frac{u^{v}}{v !}\right\}\left(\sum_{0}^{\infty} \frac{u^{v}}{v !}\right)=u e^{2 u}
$$

this proves lemma 2.

Observe that

$$
\sum_{v=0}^{\infty}(v-u) \frac{u^{v}}{v !}=0
$$

thus, if $u$ is a positive integer

$$
\begin{aligned}
& \sum_{0}^{\infty}|v-u| \frac{u^{v}}{v !}=\sum_{v \leqq u}(u-v) \frac{u^{v}}{v !}+\sum_{v \geq u}(v-u) \frac{u^{v}}{v !}= \\
& 2 \sum_{v \leqq u}(u-v) \frac{u^{v}}{v !}=2 u \sum_{v \leqq u} \frac{u^{v}}{v !}-2 u \sum_{v \leqq u-1} \frac{u^{v}}{v !}= \\
& 2 u \frac{u^{u}}{u !} \sim \frac{2 \sqrt{ } u}{\sqrt{2 \pi}} e^{u},
\end{aligned}
$$

by Stirling's formula. Thus the estimate (5) is the sharpest possible, except for a constant factor.

3. Theorem 1. Suppose that $f(x)$ is bounded in every finite interval; if $f(x)=0\left(x^{k}\right)$ for some $k>0$ as $x \rightarrow \infty$ and if $f(x)$ is continuous at a point $\zeta$, then $P(u ; f)$ converges uniformly to $f(x)$ at $x=\zeta$.

Consider

$$
\begin{aligned}
& e^{u x}\{P(u ; f)-f(x)\}=\sum_{v=0}^{\infty}\{f(v / u)-f(x)\}(u x)^{v} \frac{1}{v !}= \\
& \sum_{|v| u-x \mid \leqq \delta}+\sum_{|v / v-x|>\delta}=S_{1}+S_{2} \text {, say }
\end{aligned}
$$

and assume that $|x-\zeta|<\delta$.

Let

for

$$
\max |f(x)-f(\xi)|=m(\delta, \xi)=m(\delta),
$$

$$
|x-\xi| \leq \delta,
$$

then $m(\delta) \rightarrow 0$ as $\delta \rightarrow 0$. Now

$$
f(v / u)-f(x)=f(v / u)-f(\zeta)+f(\zeta)-f(x),
$$

and

$$
\frac{v}{u}-\zeta=\frac{v}{u}-x+x-\zeta
$$

In $S_{1}\left|\frac{v}{u}-x\right| \leqq \delta$,

hence, from eq 7

and

$$
|v / u-\zeta| \leqq 2 \delta
$$

$$
|f(v / u)-f(x)| \leqq m(2 \delta)+m(\delta) \leqq 2 m(2 \delta) .
$$

Hence

$$
\left|S_{1}\right|<2 m(2 \delta) \sum_{0}^{\infty} \frac{(u x)^{v}}{v !}=2 m(2 \delta) e^{u x} .
$$

Next write

$$
S_{2}=\sum_{v<u(x-\delta)}+\sum_{v>u(x+\delta)}=S_{3}+S_{4}, \text { say. }
$$

Then

$$
\left|S_{3}\right|<\sum_{v<u(x-\delta)} \frac{(u x)^{v}}{v !}|f(v / u)-f(x)| .
$$

Let

$$
\sup |f(x)|=U(\delta) \text {, for } x \leqq \delta
$$

Then

$$
\left|S_{3}\right|<2 U(\zeta+\delta) \sum_{u x-v>u \delta} \frac{(u x)^{v}}{v !} .
$$

Applying lemma 1 with $\lambda=u \delta$, we get

$$
\left|S_{3}\right|<2 U(\zeta+\delta) \frac{u x e^{u x}}{u^{2} \delta^{2}}=2 U(\zeta+\delta) \frac{x e^{u x}}{u \delta^{2}} .
$$

Finally, assuming $u(x+\delta)>k$,

$$
\begin{aligned}
S_{4} & =0\left(\sum_{v>u(x+\delta)} \frac{(u x)^{v}}{v !}\left(\frac{v}{u}\right)^{k}\right)=0\left(\sum_{v>u(x+\delta)} x^{k} \frac{(u x)^{v-k}}{(v-k) !}\right) \\
& =0\left(\sum_{\mu>u(x+\delta)-k} x^{k} \frac{(u x)^{\mu} \rho}{\mu !}\right) .
\end{aligned}
$$

We apply again lemma 1 , with $\lambda=u \delta-k>0$, then

$$
S_{4}=0\left(x^{k} \frac{u x e^{u x}}{(u \delta-k)^{2}}\right)=0\left(\frac{u e^{u x}}{(u \delta-k)^{2}}\right), u \rightarrow \infty .
$$

Summarizing, we find

$$
P(u ; f(x))-f(x)=0\left\{m(2 \delta)+\frac{1}{u \delta^{2}}+\frac{u}{(u \delta-k)^{2}}\right\} .
$$

Letting $u \rightarrow \infty$ for a fixed $\delta$,

$\limsup |P(u ; f(x))-f(x)| \leqq 0(m(2 \delta)), u \rightarrow \infty,|x-\zeta| \leqq \delta$,

from which our theorem follows.

It can be shown easily that uniform convergence at each point of a closed set $D$ implies uniform convergence over the set $D$. A similar argument applies to the transform (1), thus sharpening the theorems $\mathrm{A}$ and $\mathrm{B}$.

4. Theorem 2. If $f(x)$ satisfies the Lipschitztype condition 


$$
\left|f\left(x_{1}\right)-f\left(x_{2}\right)\right|<\gamma \frac{\left|x_{2}-x_{1}\right|^{\rho}}{\left(x_{1}+x_{2}\right)^{\rho / 2}}, 0<x_{1}<x_{2}<\infty,
$$

$\gamma, \rho$ constants, $0<\rho \leqq 1$,

then

$$
|P(u ; f(x))-f(x)| \leqq \gamma u^{-\rho / 2},
$$

uniformly for $0<x<\infty$, as $u \rightarrow \infty$.

We have, for $\rho=1$

$$
|P(u ; f)-f(x)| \leqq e^{-u x} \sum^{\infty}(u x)^{v} \frac{1}{v !}|f(v / u)-f(x)|
$$

$\left\langle e^{-u x} \gamma \sum_{0}^{\infty} \frac{(u x)^{v}}{v !} \frac{|v / u-x|}{(v / u+x)^{\frac{1}{2}}}=\gamma \frac{e^{-u x}}{\sqrt{u}} \sum_{0}^{\infty} \frac{(u x)^{v}}{v !} \frac{|v-u x|}{(v+u x) \frac{1}{2}}\right.$

$$
<\frac{\gamma e^{-u x}}{u \sqrt{x}} \sum_{0}^{\infty} \frac{(u x)^{v}}{v !}|v-u x| \leqq \frac{\gamma}{\sqrt{u}},
$$

by lemma 2. This proves theorem 2 for $\rho=1$. Now from Hölder's inequality, for $0<\rho<1$

$$
\begin{gathered}
e^{u x}|P(u ; f)-f(x)|=\sum_{0}^{\infty} \frac{(u x)^{v(1-\rho)}}{(v !)^{1-\rho}} \frac{(u x)^{\rho}}{(v !)^{\rho}}|f(v / u)-f(x)| \\
\left.\leqq\left(\sum_{0}^{\infty} \frac{(u x)^{v}}{v !}\right)^{1-\rho}\right)\left\{\sum \frac{(u x)^{v}}{v !}|f(v / u)-f(x)|^{1 / \rho}\right\}^{\rho} .
\end{gathered}
$$

Assume that

then

$$
\left|f\left(x_{1}\right)-f\left(x_{2}\right)\right|<\gamma \frac{\left|x_{2}-x_{1}\right|^{\rho}}{\left(x_{1}+x_{2}\right)^{\rho / 2}},
$$

$$
\begin{gathered}
e^{u x}|P(u ; f)-f(x)|<\exp ((1-\rho) u x) \gamma\left(\sum \frac{(u x)^{v}}{v !} \cdot \frac{|v / u-x|}{(v / u+x)^{1 / 2}}\right)^{\rho} \\
<\gamma \exp ((1-\rho) u x)\left(\frac{1}{u \sqrt{x}} \sqrt{u x e^{u x}}\right)^{\rho}=\gamma u^{-\rho / 2} e^{u x} .
\end{gathered}
$$

This completes the proof of theorem 2 .

Let $\rho=1$, and $f(x)=c-x$, for $0 \leqq x \leqq c, c$ a positive constant; $f(x)=0$ for $x \geqq c$. Now the condition (8) is satisfied. Furthermore

$$
P(u ; f)-f(c)=P(u ; f)=e^{-u c} \sum_{v \leqq u c} \frac{(u c)^{v}}{v !}(c-v / u)=\frac{1}{u} e^{-u c} \sum_{v \leqq u c}(u c-v) \frac{(u c)^{v}}{v !} .
$$

Let $[u c]=k$, then

and, from section 2

$$
u^{1 / 2} P(u, c)>u^{-1 / 2} e^{-k-1} \sum_{v=0}^{k}(k-v) \frac{k^{v}}{v !}
$$

$$
\sum_{n=0}^{k}(k-v) \frac{k^{v}}{v !}=\frac{k^{k+1}}{k !} \sim\left(\frac{k}{2 \pi}\right)^{3 / 2} e^{k}
$$

Thus

$$
\liminf _{u \rightarrow \infty} u^{\frac{1}{2}} P(u, c)>0 .
$$

This proves that for $\rho=1$ the order of the estimate in theorem 2 is the sharpest possible. We do not know of a similar example for $\rho<1$. For Bernstein's polynomials an exact result has been given by M. Kac [4].

5. Suppose that $f(x)$ is continuous in the infinite interval $(0, \infty)$.

Let

$$
x=\log \frac{1}{t}, 0 \leqq t \leqq 1,
$$

$f(x)=f(\log 1 / t)=\phi(t)$ is continuous in $0 \leqq t \leqq 1$.

Given $\epsilon>0$, we can find a polynomial $\sum_{i}^{n} a_{k} t^{k}=p_{n}(t)$ so that $\left|\phi(t)-p_{n}(t)\right|<\epsilon$. It follows that

$$
\left|f(x)-p_{n}\left(e^{-x}\right)\right|<\epsilon, 0<x<\infty .
$$

Now

$$
P(u ; f)=e^{-x u} \sum_{0}^{\infty} \frac{(u x)^{v}}{v !} f(v / u),
$$

and for $p_{n}\left(e^{-x}\right)$

$$
\begin{aligned}
P\left(u ; p_{n}\right) & =e^{-x u} \sum_{0}^{\infty} \frac{(u x)^{v}}{v !} \sum_{0}^{n} a_{k} e^{-k v / u} \\
& =e^{-x u} \sum_{0}^{n} a_{k} \sum_{0}^{\infty} \frac{(u x \exp (-k / u))^{v}}{v !} \\
& =e^{-x u} \sum_{0}^{n} a_{k} \exp (u x \exp (-k / u)) \\
& =\sum_{0}^{n} a_{k} e^{-u x(1-\exp -k / u)} .
\end{aligned}
$$

Clearly for $u \rightarrow \infty, P\left(u ; p_{n}\right) \rightarrow p_{n}\left(e^{-x}\right)$ uniformly in $(0, \infty)$; furthermore

$$
f(x)=p_{n}\left(e^{-x}\right)+\epsilon_{n}(x),\left|\boldsymbol{\epsilon}_{n}(x)\right|<\epsilon
$$


and

$$
P(u ; f)=P\left(u, f-p_{n}\right)+P\left(u, p_{n}\right) .
$$

Here

$$
\left|P\left(u ; f-p_{n}\right)\right|<\epsilon,
$$

hence

$$
\begin{gathered}
|P(u ; f)-f(x)|<\epsilon+\left|P\left(u ; p_{n}\right)-f(x)\right| \leqq \epsilon+ \\
\left|P\left(u ; p_{n}\right)-p_{n}\left(e^{-x}\right)\right|+\left|p_{n}\left(e^{-x}\right)-f(x)\right| .
\end{gathered}
$$

Thus, the theorem:

Theorem 3. If $f(x)$ is continuous in $(0, \infty)$ then $P(u ; f) \rightarrow f(x)$ uniformly in $(0, \infty)$.

6. Theorem 4. If $f(x)$ is $r$-times differentiable, $f^{(r)}(x)=0\left(x^{k}\right)$ as $x \rightarrow \infty$, for some $K>0$, and if $f^{(r)}(x)$ is continuous at a point $\zeta$, then $P^{(r)}(u ; f)$ converges uniformly to $f^{(\tau)}(x)$ at $x=\zeta$.

We write $1 / u=h$, and introduce the notation

$$
\begin{aligned}
\Delta f(v h) & =f(\overline{v+1} h)-f(v h) . \\
\Delta^{2} f(v h) & =\Delta \Delta f(v h)=f(\overline{v+2} h)-2 f(\overline{v+1} h)+f(v h) . \\
\Delta^{r} f(v h) & =\Delta \Delta^{r-1} f(v h) \\
& =\sum_{k=0}^{r}(-1)^{k}\left(\begin{array}{l}
r \\
k
\end{array}\right) f(\overline{v+k} h), r \geqq 0 .
\end{aligned}
$$

$P(1 / h ; f)=Q(h ; f)=e^{-x / h} \sum_{v=0}^{\infty} 1 / v !\left(\frac{x}{h}\right)^{v} f(v h)$.

Lemma 3. We have

$$
e^{x / h} \frac{d^{r}}{d x^{r}} Q(h ; f)=\sum_{0}^{\infty} \Delta^{r} f(v h) 1 / v !\left(\frac{x}{h}\right)^{v} h^{-r} .
$$

Differentiation gives

$$
\begin{aligned}
\frac{d}{d x} Q(h ; f)= & e^{-x / h} \sum_{0}^{\infty} \frac{1}{(v-1) !}\left(\frac{x}{h}\right)^{v-1} \frac{1}{h} f(v h)- \\
& \frac{1}{h} e^{-x / h} \sum_{0}^{\infty} \frac{1}{v !}\left(\frac{x}{h}\right)^{v} f(v h) \\
= & \frac{1}{h} e^{-x / h} \sum_{0}^{\infty} \frac{1}{v !}\left(\frac{x}{h}\right)^{v} \Delta f(v h) .
\end{aligned}
$$

The lemma now follows by induction.

It is known that

$$
\frac{\Delta^{r} f(v h)}{h^{r}}=f^{(r)}(\eta)
$$

where

$$
v h<\eta<(v+r) h .
$$

Now

$$
\begin{aligned}
& D_{r} Q(h ; f)-f^{(r)}(x) \\
& =e^{-x / h} \sum_{0}^{\infty}\left\{\frac{h^{-r} \Delta^{r} f(v h)-f^{(r)}(x)}{v !}\right\}\left(\frac{x}{h}\right)^{v} \\
& =e^{-x / h}\left\{\sum_{|v h-x| \leqq \delta}+\sum_{|v h-x|>\delta}\right\},
\end{aligned}
$$

where we assume that $|x-\zeta|<\delta$. Using the same device as in the proof of theorem 1, we get theorem 4. For Bernstein's polynomials see G. Lorentz [5], and his reference to Wigert's work.

For $\rho=1$ theorem 2 and formula (6) suggest the following proposition:

Theorem 5. If $f(x)$ is bounded in every finite interval, if it is differentiable at a point $\zeta>0$, and if $f(x)=0\left(x^{k}\right)$ for some $k>0, x \rightarrow \infty$, then

Let

$$
u^{3 / 2}\{P(u ; f(\zeta))-f(\zeta)\} \rightarrow 0, u \rightarrow \infty .
$$

$$
\max \left|\frac{f(\zeta+h)-f(\zeta)}{h}-f^{\prime}(\zeta)\right|=\mu(\delta, \zeta)=\mu(\delta),
$$

then $\mu(\delta) \rightarrow 0$ as $\delta \rightarrow 0$. We may write

where

$$
f(\zeta+h)-f(\zeta)=h f^{\prime}(\zeta)+h \epsilon(\zeta, h),
$$

Now

$$
|\epsilon(\zeta, h)| \leqq \mu(\delta) \text { for }|h| \leqq \delta
$$

$$
P(u ; f(\zeta))-f(\zeta)=e^{-u \zeta} \sum_{0}^{\infty} \frac{(u \zeta)^{v}}{v !}\left\{\left(\frac{v}{u}-\zeta\right) f^{\prime}(\zeta)+\right.
$$

where

$$
\left.\left(\frac{v}{u}-\zeta\right) \epsilon_{v}(u)\right\}
$$

$$
\left|\epsilon_{v}(u)\right| \leqq \mu(\delta) \text { for }\left|\frac{v}{u}-\zeta\right| \leqq \delta .
$$

Utilizing formula (6) we get

$$
\begin{aligned}
P(u ; f)-f(\zeta) & =\frac{1}{u} e^{-u \zeta} \sum_{0}^{\infty} \frac{(u \zeta)^{v}}{v !}(v-u \zeta) \epsilon_{v}(u) \\
& =\frac{1}{u} e^{-u \zeta}\left\{\sum_{|v-u \zeta| \leqq \delta u}+\sum_{|v-u \zeta|>\delta u}\right\} .
\end{aligned}
$$

Using the same device as in the proof of theorem 1 , and employing lemma 2 , we can complete the proof of theorem 3 .

The result can be generalized to higher derivatives. We restrict ourselves here to the case that $f^{\prime \prime}(\zeta)$ exists. Thus,

$$
f(\zeta+h)-f(\zeta)=h f^{\prime}(\zeta)+\frac{1}{2} h^{2}\left\{f^{\prime \prime}(\zeta)+\epsilon(\zeta, h)\right\},
$$


where

$|\epsilon(\zeta, h)| \leqq \eta(\delta)$ for $|h| \leqq \delta$, and $\eta(\delta) \rightarrow 0, \delta \rightarrow 0$.

Now

$$
\begin{aligned}
& P\{u ; f(\zeta)\}-f(\zeta)=e^{-u \zeta} \sum_{0}^{\infty} \frac{(u \zeta)^{v}}{v !}\left\{\left(\frac{v}{u}-\zeta\right) f^{\prime}(\zeta)+\right. \\
& \left.\frac{1}{2}\left(\frac{v}{u}-\zeta\right)^{2} f^{\prime \prime}(\zeta)\right\}+e^{-u \zeta} \sum_{0}^{\infty} \frac{(u \zeta)^{v}}{v !} \frac{1}{2}(v / u-\zeta)^{2} \epsilon_{v}(u),
\end{aligned}
$$

where

$$
\left|\epsilon_{v}(u)\right| \leqq \eta(\delta) \text { for }\left|\frac{v}{u}-\zeta\right| \leq \delta .
$$

It follows from formulas 4 and 6 that

$$
\begin{aligned}
P(u ; f(\zeta))-f(\zeta)=\frac{\zeta}{2 u} f^{\prime \prime}(\zeta)+ & \\
& \frac{e^{-u \zeta}}{2 u^{2}} \sum_{0}^{\infty} \frac{(u \zeta)^{v}}{v !}(v-u \zeta)^{2} \epsilon_{v}(u),
\end{aligned}
$$

or

$$
\begin{aligned}
u\{P(u ; f)-f(\zeta)\}= & \frac{1}{2} \zeta f^{\prime \prime}(\zeta)+ \\
& \frac{e^{-u \zeta}}{2 u} \sum_{0}^{\infty} \frac{(u \zeta)^{v}}{v !}(v-u \zeta)^{2} \epsilon_{v}(u) .
\end{aligned}
$$

We write

$$
\begin{aligned}
\sum_{0}^{\infty} \frac{(u \zeta)^{v}}{v !}(v-u \zeta)^{2} \epsilon_{v}(u) & =\sum_{|v-u \xi| \leqq u \delta}+\sum_{|v-u \xi|>u \dot{\delta}} \\
& =T_{1}+T_{2}, \text { say. }
\end{aligned}
$$

From (9) and (4),

$$
\left|T_{1}\right|<\eta(\delta) u \zeta e^{\mu \zeta} .
$$

Hence,

$$
\frac{e^{-u \zeta}}{2 u}\left|T_{1}\right|<\frac{1}{2} \eta(\delta) \zeta
$$

Next write

$$
T_{2}=\sum_{v<u(\zeta-\delta)}+\sum_{v>u(\zeta+\delta)}=T_{3}+T_{4}, \text { say }
$$

and note that

$$
\begin{aligned}
\frac{1}{2}\left(\frac{v}{u}-\zeta\right)^{2} \epsilon_{v}(u)= & f\left(\frac{v}{u}\right)-f(\zeta)-(v / u-\zeta) f^{\prime}(\zeta)- \\
& \frac{1}{2}\left(\frac{v}{u}-\zeta\right)^{2} f^{\prime \prime}(\zeta)
\end{aligned}
$$

Let

$$
\sup |f(x)|=M(\zeta), x \leqq \zeta
$$

then

$$
\begin{aligned}
\left|T_{3}\right|<\left\{2 M(\zeta)+\zeta\left|f^{\prime}(\zeta)\right|+\frac{1}{2} \zeta^{2}\left|f^{\prime \prime}(\zeta)\right|\right\} & \\
& \sum_{u \zeta-v>u \delta} u^{2} \xi^{2} \frac{1}{v !}(u \zeta)^{\bullet},
\end{aligned}
$$

We now employ the formula (see e.g. [2], p. 200)

$$
\sum_{|r-u|>\delta u} e^{-u} \frac{u^{v}}{v !}=0\left(\exp \left(-\frac{1}{3} \delta^{2} u\right)\right), u \rightarrow \infty
$$

It follows that

$$
e^{-u \zeta} \sum_{u \zeta-v>u \delta} \frac{(u \zeta)^{v}}{v !}=0\left(\exp \left(-\frac{1}{3} \frac{\delta^{2}}{\zeta} u\right)\right),
$$

so that

$$
\frac{e^{-u \zeta}}{u} T_{3}=0\left\{u \exp \left(-\frac{1}{3} \frac{\delta^{2}}{\zeta} u\right)\right\} .
$$

Finally, in view of $f(x)=0\left(x^{k}\right)$, we have for $v>u \zeta$ and $k \geq 2$

$$
(v-u \zeta)^{2} \boldsymbol{\epsilon}_{v}(u)=0\left(u^{2} \frac{v^{k}}{u^{k}}\right)
$$

hence

$$
\begin{aligned}
T_{4} & =0\left(\sum_{v-u \zeta>u \delta} \frac{(u \zeta)^{v}}{v !} \frac{v^{k}}{u^{k}}\right)=0\left(\sum_{v-u \zeta>u \delta} u^{2} \frac{(u \zeta)^{v-k}}{(v-k) !}\right) \\
& =0\left(\sum_{v-u \zeta>u \delta-k} u^{2} \frac{(u \zeta)^{v}}{v !}\right)=0\left\{u^{2} \exp \left(u \zeta-\frac{\delta^{2}}{3 \zeta} u\right)\right\} .
\end{aligned}
$$

Thus,

$$
\frac{e^{-u \zeta}}{u} T_{4}=0\left\{u \exp \left(-\frac{1}{3 \zeta} \delta^{2} u\right)\right\}
$$

Summarizing, from (10) and (11),

$$
\lim \sup \mid u\left\{P(u ; f(\zeta)-f(\zeta)\}-\frac{1}{2} \zeta f^{\prime \prime}(\zeta) \mid \leqq \delta .\right.
$$

But $\delta$ is arbitrarily small, hence the theorem:

Theorem 6. If $f(x)$ is bounded in every finite interval, if it is twice differentiable at a point $\zeta>0$, and if for some $k>0 f(x)=0\left(x^{k}\right), x \rightarrow \infty$, then

$$
u\{P(u ; f(\zeta))-f(\zeta)\} \rightarrow \frac{1}{2} \zeta f^{\prime \prime}(\zeta), u \rightarrow \infty .
$$

Analogous theorems for Bernstein's polynomials were given in [1] and [8].

8. In the terminology of probability distribution the Bernstein polynomial corresponds to the binomial distribution. The distribution function is

$$
\sum_{v \leqq r}\left(\begin{array}{l}
n \\
v
\end{array}\right) t^{v}(1-t)^{n-v}=F_{n}(r)
$$


the linear functional $B_{n}(t ; f)$ is

$$
\int_{0}^{1} f(r / n) d F_{n}(r)=\sum_{v=0}^{n} f(v / n)\left(\begin{array}{l}
n \\
v
\end{array}\right) t^{v}(1-t)^{n-v} .
$$

Similarly, $P(u ; f)$ corresponds to the Poisson distribution; the distribution function is

$$
\sum_{v \leqq r} \frac{x^{v}}{v !} e^{-x}=G(r, x)
$$

and

$P(u ; f)=\int_{0}^{\infty} f(r / u) d G(r, u x)=\sum_{v=0}^{\infty} e^{-u x} \frac{(u x)^{v}}{v !} f(v / u)$

here the term with the largest weight has the index $v \sim u x$. In the Bernoulli polynomial the term with the largest weight has the index $v \sim t n$.

If instead of a function $f$ we consider a sequence $S_{0}, S_{1}, S_{2}, \ldots$, then to the transform (1) corresponds

$$
\sum_{v=0}^{n}\left(\begin{array}{l}
n \\
v
\end{array}\right) t^{v}(1-t)^{n-v} S_{v}, n \rightarrow \infty,
$$

which defines the generalized Euler summability, and to the transform (2) corresponds

$$
e^{-x} \sum_{v=0}^{\infty} \frac{x^{v}}{v !} S_{v}, x \rightarrow \infty,
$$

which gives Borel's summability method.

9. To approximate a function $f(x)$ over the whole real axis, we write

$f(x)=\frac{f(x)+f(-x)}{2}+\frac{f(x)-f(-x)}{2}=f_{1}(x)+f_{2}(x)$, say $;$

obviously

$$
f_{1}(-x)=f_{1}(x), f_{2}(-x)=-f_{2}(x) .
$$

Now

$$
P(u ; f)=P\left(u ; f_{1}\right)+P\left(u ; f_{2}\right) \text {; }
$$

here

$$
P\left(u ; f_{1}\right)=e^{-u x} \sum_{0}^{\infty} \frac{(u x)^{v}}{v !} f_{1}(v / u),
$$

and if we change $x$ into $-x, u$ into $-u$, we get

$$
P\left(-u ; f_{1}(-x)\right)=P\left(u ; f_{1}(x)\right),
$$

so that our previous results are directly applicable. Similarly,

$$
P\left(-u ; f_{2}(-x)\right)=-P\left(u ; f_{2}(x)\right) ;
$$

thus for negative values of $x$ we need only change $u$ into $-u$, and revert to our previous results.

10. It follows from a well-known property of the Beta function that

$$
\left(\begin{array}{l}
n \\
v
\end{array}\right) \int_{0}^{1} t^{v}(1-t)^{n-v} d t=\frac{1}{n+1}, v=0,1, \cdots, n ;
$$

hence

$$
\int_{0}^{1} B_{n}(t) d t=\frac{1}{n+1} \sum_{v=0}^{n} f(v / n),
$$

so that for any Riemann integrable function

$$
\int_{0}^{1} B_{n}(t) d t \rightarrow \int_{0}^{1} f(t) d t .
$$

Similarly, at first formally

$$
\begin{aligned}
& \int_{0}^{\infty} P(u ; f) d x=\sum_{0}^{\infty} \frac{u^{v}}{v !} f(v / u) \int_{0}^{\infty} e^{-x u} x^{v} d x= \\
& \frac{1}{u} \sum_{0}^{\infty} f(v / u) ;
\end{aligned}
$$

the interchange of integration and summation is legitimate if the series $\sum f(v / u)$ is convergent. Thus, the formula

$$
\int_{0}^{\infty} P(u ; f) d x=\frac{1}{u} \sum_{0}^{\infty} f(v / u)
$$

is valid if both sides exist. However, it is a delicate question under what conditions

$$
\lim _{u \rightarrow \infty} \frac{1}{u} \sum_{0}^{\infty} f(v / u) \rightarrow \int_{0}^{\infty} f(x) d x
$$

An extensive literature deals with this question.

\section{References}

[1] S. Bernstein, Complément à l'article de E. Voronovskaja, Détermination de la forme, etc. Compt. Rend. l'acad. sci. URSS, 4, 86 to 92 (1932).

[2] G. H. Hardy, Divergent series (Oxford Univ. Press., 1949).

[3] F. Herzog and J. D. Hill, The Bernstein polynomials for discontinuous functions, Am. J. Math., 68, 109 to 124 (1946).

[4] M. Kac, Une remarque sur les polynomes de M. S. Bernstein, Studia Mathematica, $\boldsymbol{\gamma}, 49$ to 51, 8, 170 (1938).

[5] G. Lorentz, Zur theorie der polynome von S. Bernstein, Mathematicheskii Sbornik (Recuil Mathematique), Nouvelle Serie, 2, (44) 543 to 556 (1937).

[6] G. Pólya and G. Szegö, Aufgaben und Lehrsätze aus der Analysis, 1, Berlin (1925). 
[7] T. Popoviciu, Sur l'approximation de functions convexes d'ordre superieur, Mathematica, 10, 49 to 54 (1935).

[8] E. Voronovskaja, Détermination de la forme asymptotique de l'approximation des fonctions par les polynômes de S. Bernstein, Compt. Rend. l' acad. sci. URSS, 4, 79 to 85 (1932).

Attention is called to relevant work of Einar Hille (see

Proc. Nat. Acad. Sci., p. 421 to 424 (Oct. 28, 1942), and his book: Functional analysis and semi-groups, Am. Math. Soc. Colloqu. publications 31, 186 to 189 , 323 to 325,332 to 333 (1948), where transform (2) is dealt with as a special case of a more general transformation. It contains in particular some part of the results of this paper.

Los Angeles, November 9, 1949.

\title{
Numerical Determination of Characteristic Numbers ${ }^{1}$
}

\author{
By W. E. Milne
}

\begin{abstract}
This paper represents a contribution to the problem of characteristic values and characteristic solutions of ordinary linear differential equations. The problem is conceived as a vibration problem in $x$ and $t$. The partial differential equation is then approximated by a difference equation in both entries. The problem is now to find those frequencies in which a separation in $x$ and $t$ takes place. This is done by finding the roots of a trigonometric expansion of certain order. The method is applied to a number of interesting cases that illustrate various types of situations encountered in problems of physics and engineering.
\end{abstract}

An improved method for obtaining the latent roots of a matrix has been devised by Cornelius Lanczos of this Bureau. He has shown further how this process may be used to secure the characteristic numbers (eigenwerte) belonging to a boundary value problem associated with an ordinary linear differential equation. The purpose of this note is to present a procedure for calculating characteristic numbers, based essentially on this method of Lanczos, but modified in such a way as to provide a simple numerical routine for the computation. A number of numerical examples, worked in full, illustrate the procedure and give an indication of the accuracy attained. The exposition is limited to the case of differential equations of the second order, but the method is capable of extension to cases of higher order.

1. The problem. Let

$$
L(u)=P_{0}(x) \frac{d^{2} u}{d x^{2}}+P_{1}(x) \frac{d u}{d x}+P_{2}(x) u .
$$

The problem before us is to find those characteristic values of $\lambda$ for which the differential system

$$
\begin{aligned}
& L(u)+\lambda^{2} u==0 \\
& \left.\begin{array}{l}
u+g \frac{d u}{d x}=0 \text { at } x=a \\
u+G \frac{d u}{d x}=0 \text { at } x=b
\end{array}\right\}
\end{aligned}
$$

possesses nonzero solutions in the interval $a \leqq x \leqq b$.

Problems of this type arise in many different ways in mathematical physics. For example, they may occur in connection with the heat equation

$$
\frac{\partial V}{\partial t}=L(V)
$$

if we assume particular solutions of the form

$$
V=u(x) e^{-\lambda^{2} t},
$$

or in connection with the wave equation

$$
\frac{\partial^{2} V}{\partial t^{2}}=L(V)
$$

1 The preparation of this paper was sponsored (in part) by the Office of Naval Research. 